\title{
Location Models for Ceding Market Share and Shrinking Services
}

\author{
Charles ReVelle ${ }^{1}$
}

Alan T. Murray ${ }^{2}$

Daniel Serra ${ }^{3}$

\begin{abstract}
New location models are presented here for exploring the reduction of facilities in a region. The first of these models considers firms ceding market share to competitors under situations of financial exigency. The goal of this model is to cede the least market share, i.e., retain as much of the customer base as possible while shedding costly outlets. The second model considers a firm essentially without competition that must shrink it services for economic reasons. This firm is assumed to close outlets so that the degradation of service is limited. An example is offered within a competitive environment to demonstrate the usefulness of this modeling approach.
\end{abstract}

Keywords: de-location, shrinkage, covering models.

JEL Codes: C61, J80

\footnotetext{
${ }^{1}$ The Johns Hopkins University

${ }^{2}$ Ohio State University

${ }^{3}$ Universitat Pompeu Fabra. Research funded by MCYT, Project SEC2003-1991, Spain.
} 


\section{Introduction}

Location science, with its components of theory and modeling, continues to grow dramatically in the disciplinary areas of geography, management, mathematics, economics and operations research. In general, the purpose of this research field is to formulate quantitative models to site a given set of facilities in a region. The region can be represented by a network, continuous space, or can be a set of discrete points. There are models for locating public facilities such as schools or post-offices, models for locating retail facilities in the presence of competition, models for locating emergency services such as fire stations or ambulances, and models for locating plants and warehouses, among others. These quantitative models can be formulated and solved using linear programming, integer programming, dynamic programming or by heuristic or metaheuristic approaches. Facilities may have capacity restrictions, experience congestion, have minimum thresholds for level of service and other defining characteristics. The demand to be served may be deterministic or stochastic to address uncertainty issues. Thus, a myriad of formulations are available within the field of location science to address very different issues related to the formulation, solution and implementation of location problems. An overview of the state of the art in this area is presented in Drezner (2003). But most location models (if not all) have one thing in common: they are proactive, in that they all seek the location of new facilities, or they are reactive, in that they seek to relocate and open new locations at the same time. The literature has paid little attention to the fact that sometimes it is necessary to close facilities (Wang et al. 2003).

In the private sector, the marketplace for contemporary industrial and service goods is often highly competitive. Firms map out their activities in light of market forces and the action of rival firms in the industry. Changes in the competitive structure of the industry call for new strategies. Strategies do not always succeed as planned, however; plant or store closure may occur as an integral part of strategy or in spite of it. Corporate planners often fail to predict future market trends or are unable to maintain their market share when competitive conditions in the industry change. They may not perceive the gravity of market changes, or they may believe that their established market position leaves them immune to market dynamics. In other cases, production systems do not operate as planned and products fail to meet consumer expectations. As well, new products may be introduced. All these conditions may lead to rivals succeeding in stealing market share. Thus changes in the competitive structure of industries and services may lead to plant or store closures in two distinct ways: as an integral part of the process of strategic adaptation or as a consequence of failure to adapt to new industry conditions.

As an example, Plant Closing News, a biweekly, industry-focused newsletter (see www.plantclosings.com), found that only in the first week of 2003 there were more than 500 ind ustrial plant closures in the U.S. and Canada, in very diverse industrial sectors (Table 1). Plant closings are the most visible manifestation of market dynamics and corporate restructuring on the economic landscape. 
Table 1: Plant Closure in USA and Canada, 2/1/03-7/1/03

\begin{tabular}{|l|c|}
\hline Industry & Number of Plant Closings \\
\hline Food & 74 \\
Chemical & 40 \\
Cigarettes & 1 \\
Refined oil products & 7 \\
Textiles & 23 \\
Plastics & 48 \\
Wood products & 30 \\
Glass, cement & 8 \\
Pulp and paper & 47 \\
Metal products & 163 \\
Chemicals & 40 \\
Electronics & 72 \\
Other & 7 \\
Total & $\mathbf{5 2 0}$ \\
\hline
\end{tabular}

Another reason for plant closure is related to business cycles. When the economy is in a growing period, demand for products and services is high, leading to new investments and the building of new facilities. However, when the business cycle is in recession, some investments are no longer profitable and it becomes necessary to close some of the currently operating facilities in order to survive. In Figure 1, the growth rate for the U.S. from 1970 to 2003 clearly shows the roller-coaster ups and downs of the economy. The shaded areas represent periods of economic crises, with plant closures, massive layoffs and demand downfall. In these periods firms need to downsize their capacity and infrastructure to an appropriate size in order to cope with the economic situation.

Another argument for plant closures is based on the fact that some economic sectors and industries are in decline. A declining industry is defined as an industry group's employment level decreasing for two quarters by five percent or more over the year In fact, a significant fraction of U.S. manufacturing output is accounted for by declining industries. In these industries, the important competitive moves pertain to disinvestments rather than investment. Capacity must be reduced in order to restore profitability Capacity reduction is, however, like a public good; each firm would prefer that its competitors shoulder the reduction. 


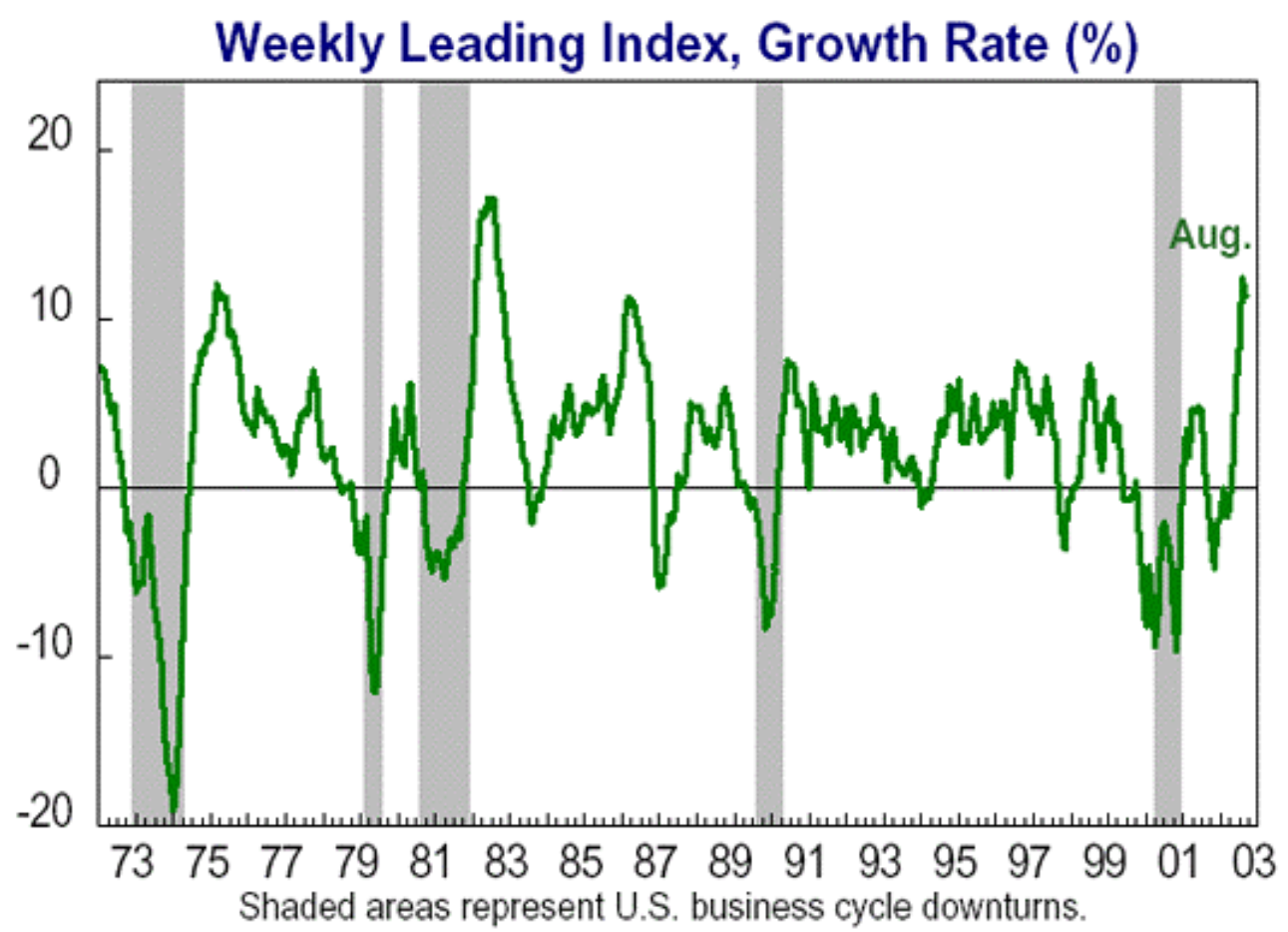

Figure 1. U.S. business for 1970 to 2003

(Source: www.businesscycle.com)

Some plant location models have been developed to address the issue of plant closure in a multiperiod setting. Klincewicz et al. (1988) formulated a large-scale multilocation capacity-planning model. The model chooses a multiperiod schedule of openings, expansions, and closings of facilities and assigns demand locations to these facilities. Although generic in nature, this model was developed to plan the evolution of material logistics systems over time. In order to have a truly practical tool, numerous features are considered including existing configuration, arbitrary demand patterns, concave operating costs, single-source assignments, demand location reassignment costs, and others.

A real-world application involving the relocation and phase-out of a combined manufacturing plant and warehousing facility was presented by Melachrinoudis and Min (2000). The relocation and phase-out decision was called for to adapt to dynamic changes in business environments surrounding the firm's supply chain operations. Such changes included changes in supplier and customer bases, distribution networks, corporate re-engineering, business climate, and government legislation. To aid management in formulating a more effective relocation strategy, Melachrinoudis and Min (2000) assess the viability of a proposed site from multi-echelon supply chain perspectives and determine the optimal timing of relocation and phase-out in the planning horizon using a dynamic, multiple objective, mixed-integer programming model.

Wang et al. (2003) studied a budget constrained location problem in which they simultaneously consider opening some new facilities and closing some existing facilities. The objective is to minimize the total weighted travel distance for customers subject to a constraint on the budget for opening and/or closing facilities and a constraint on the total number of open facilities desired. Their application focused on 
locating/relocating bank branches in Amherst, New York. They also discuss the situation where operating costs are part of the objective function.

The retailing sector is also affected by business downturns that can cause the closure of many (even hundreds of) retail stores. For example, a serious economic crisis at KMart Corporation forced the company to close many of their stores across U.S. after years of struggling against more powerful and successful rivals Wal-Mart Stores Inc. and Target Corp. One chain, Ames, closed all of its stores. In order to ensure a more competitive position and to exit from bankruptcy, K-Mart closed more than $30 \%$ of its stores across the United States (Schenke 2002).

Facility closing is also a common feature in the public sector. For example, on April 14, 1993 the Minister of Health of the Province of Saskatchewan announced the closure of 52 of the 112 small hospitals in the Province based on: size, utilization for two consecutive years and distance to the nearest-neighboring hospital (Lepnurm and Lepnurm 2003).

Frederick and Weston (1982) formulated a model to find the optimal location of telephone answering sites for a non-competitive service organization within the public sector. The methodological approach taken to addressing the telephone site location problem was integer programming. The modelling effort resulted in several organization changes including opening and closing of individual offices, domain realignments of new or existing offices, and significant cost savings primarily due to telecommunication cost reductions and increased utilization of telephone answering manpower.

Perhaps the most significant modelling work in the public sector has been for school system consolidation as a result of declining enrolments. Noteworthy initial efforts in this area were Bruno and Anderson (1982) and Diamond and Wright (1987). The work of Diamond and Wright (1987) was unique in that balancing utilization was also a goal. Church and Murray (1993) also examine consolidation combined with balancing utilization.

Other instances where it may be necessary to close facilities are related to public transport. For example, in urban bus transportation it may be necessary to eliminate some stops and relocate others due to changes in population density and network changes. There is, on the one hand, a trade off between number of stops and geographical coverage, with more stops providing greater coverage. On the other hand, more stops along a route translate to greater service interruption and longer travel times. Murray and Wu (2002) study some modelling approaches for addressing accessibility concerns in an integrated fashion. They illustrate the models using busbased transit in Columbus, Ohio.

In this paper we formulate the Planned Shrinkage Problem, a general model to close facilities. We present the general model in the next section. In the third section, computational experience is recounted in the context of a specific example. Finally, some conclusions are offered.

\section{Problem formulation}




\section{A. Firm with competition}

The Planned Shrinkage Problem can be formulated in a competitive setting. Let us consider a spatial market that is represented by discrete points in a connected network. Each node can represent a local market and has a parameter that can represent population or local demand for the product that is being offered. On the other hand, some - but not all- of the nodes in the network have servers that offer the product. These servers belong to different firms that compete for consumers. Each of these firms can have more than one server located somewhere in the system. As a condition of the problem statement, the product that is sold is homogeneous, and its price is the same everywhere in the market. Further, it is assumed that all firms bear the same unit costs. Consumers will travel to their closest facility to obtain the desired product. A firm captures a consumer node if one of its servers is closer to that node than any of its competitors' servers.

Suppose that a firm (say firm A) is operating in the market with $q$ outlets. Due to market conditions (for example, increase in competition and/or decline in demand), Firm A wishes to retain $p$ of its $q$ outlets with the objective of minimizing the impact on demand loss to competitors. The model can be reformulated as follows:

\section{Parameters:}

$i=$ index of nodal areas to be served

$j=$ index of existing facilities from all firms operating in the market

$a_{i}=$ user population or demand in nodal area $i$

$d_{i j}=$ shortest distance or travel time from area $i$ to outlet $j$

$\bar{d}_{i}=$ distance from area $i$ to closest competitor $=\min _{j} d_{i j}$

$M_{i}=\left\{j \mid d_{i j}<\bar{d}_{i}\right\}=$ the set of servers that are closer to $i$ than A's nearest competitor

$n=$ number of demand nodes

$p=$ number of facilities retained

Decision variables:

$y_{i}= \begin{cases}1 & \text { if demand at } i \text { that is lost to competitor } \mathrm{s} \\ 0 & \text { otherwise. }\end{cases}$

$x_{j}= \begin{cases}1 & \text { if outlet } j \text { is retained } \\ 0 & \text { otherwise }\end{cases}$

The mathematical formulation of the problem is as follows:

Minimize

$$
Z=\sum_{i} a_{i} y_{i}
$$

Subject to 


$$
\begin{gathered}
y_{i} \geq 1-\sum_{j \in M_{i}} x_{j} \quad \forall i \\
(2) \\
\sum_{j} x_{j}=p \\
(3) \\
y_{i}=(0,1) \quad \forall i \\
(4) \quad \forall j \\
x_{j}=(0,1) \quad \forall j
\end{gathered}
$$

The model minimizes the demand lost to competitors due to the closing of some of its existing outlets. Constraint set (2) works as follows: if there is a Firm A facility closer to node $i$ than the closest competitor, the node is not lost to the competitor. On the other hand, if $\sum_{j \in M_{i}} x_{j}=0$ then $y_{i}=1$ and the demand at $i$ is lost to the competitor, since there is no outlet of Firm A that is closer to $i$ than any other competitor outlet. Observe that the set $M_{i}$ is defined so that, if there is a tie in distance between Firm A and the competitors to a given node, the demand is not lost, since we only consider Firm A outlets that are closer to $i$ than the closest competitor. If previous to the model we assume that in case of ties, demand is split among competitors, the use of the Planned Shrinkage Model as formulated will not affect these ties.

This model may be regarded as an inverse formulation of the Maximum Capture Location Problem formulated by ReVelle (1986). In the Maximum Capture model, a new firm wants to enter a market with a given set of outlets. In the above developed (NAME ?) model, a firm is decreasing its presence but wants to do this with the least loss of market share.

The formulation may also be written as follows. Besides all the original variables and parameters, we introduce:

$y_{i}=1,0 ; 1$ if demand node $\mathrm{i}$ is retained by the firm, and 0 otherwise.

Therefore, $y_{i}=1-y_{i}$

The objective (1) may be rewritten as:

$$
\text { Minimize } \quad z=\sum_{i \in I} a_{i} y_{i}=\sum a i\left(1-y_{i}\right)
$$

This is equivalent to:

$$
\text { Maximize } z=\sum a i \mathrm{y}_{\mathrm{i}}
$$

That is, maximizing demand retained when eliminating facilities. Constraint (2) would then be:

$$
1-y_{i} \geq 1-\sum_{j \in M i} x_{j}
$$


This simplifies to:

$$
y_{i} \leq \sum_{j \in M i} x_{j} \quad i=1,2, \ldots, m
$$

These derived constraints, combined with the above objective, are equivalent to maximizing the demand retained when closing facilities, where demand is retained only if one or more facilities are maintained in the set $M_{i}$,. That is, one or more facilities are maintained in positions closer than the nearest competitor to $i$. Given this, the new formulation is equivalent to the Maximal Covering Location Problem (MCLP) detailed in Church and ReVelle (1974).

\section{B. Firm without competition}

Suppose now a firm that is operating in that market essentially without competition (perhaps it is a public service such as the post office) with several retail or service outlets. The firm wishes to close some of these outlets to decrease costs. The objective is to minimize the number of people made worse-off or to minimize some system measure of deterioration of service.

The additional notation is as follows:

$\bar{d}_{i}=$ distance from area $i$ to closest facility $=\min _{j} d_{i j}$

$\beta=$ threshold for nearest facilities (e.g., 25\%)

$M_{i}=\left\{j \mid d_{i j} \leq \beta \bar{d}_{i}\right\}=$ the set of servers $j$ that are within a factor 1.25 times the current distance from $i$ to its closest facility

$S=$ maximum service distance or travel time (a level of service that will not be violated)

$$
N_{i}=\left\{j \mid d_{i j} \leq S\right\}
$$

Decision variables:

$y_{i}= \begin{cases}1 & \text { if demand at } i \text { is made worse off by facility closures } \\ 0 & \text { otherwise. }\end{cases}$

$x_{j}= \begin{cases}1 & \text { if facilty } j \text { is retained } \\ 0 & \text { otherwise. }\end{cases}$

The mathematical formulation of the problem is as follows:

Minimize

$$
Z=\sum_{i} a_{i} y_{i}
$$


Subject to

$$
\begin{gathered}
\sum_{j \in N_{i}} x_{j} \geq 1 \quad \forall i \\
y_{i} \geq 1-\sum_{j \in M_{i}}^{(6)} x_{j} \quad \forall i \\
\sum_{j} x_{j}=p
\end{gathered}
$$

$$
\begin{aligned}
y_{i}=(0,1) \quad \forall i \\
(9) \\
x_{j}=(0,1) \quad \forall j
\end{aligned}
$$

The objective minimizes the population that will be uncovered within a threshold level after the closing of $p$ facilities. Thus, the first constraint guarantees that all the population will be covered within that distance standard. In order to limit how much worse off an area becomes, constraint (7) is utilized to prevent coverage deterioration beyond a threshold level $S$. The second constraint accounts for population loss at node $i$ if there is no facility within $\beta \bar{d}_{i}$. The use of $\beta$ allows for a range of "nearest" facilities to be modeled. If $\beta=1$, then the set $M_{i}$ will consist of the nearest facility $j$ to area $i$ or the nearest facilities if there is a tie. Alternatively if $\beta=1.25$, as an example, then the nearest set will consist of those facilities at most $25 \%$ farther than the closest facility. Finally, the last constraint sets the number of outlets to be retained.

\section{Application Results}

The competitive version of the shrinkage problem was tested using the well-known 55-node network of Swain, see appendix. The demand at each node is indicated in the appendix. The total amount of demand to be captured is equal to 3,575. The model was solved using LINGO version 6.

In this example, eight outlets are being operated by two firms (say Firm A and Firm B), 4 outlets each. Firm A wants to reduce the number of outlets and therefore wants to know which ones have to be removed so as to minimize the impact on capture reduction.

In order to proceed, the initial locations for Firm A were found using the Maximum Covering Location Problem (Church and ReVelle 1974) with a maximum coverage distance set to 100 . Then, the location of the 4 outlets for Firm B were determined using the Maximum Capture Location Problem (ReVelle 1986).

Table 1: Results, 55-node network

\begin{tabular}{|l|l|l|l|}
\hline Number of outlets & Firm A & $\%$ & Firm B \\
\hline
\end{tabular}




\begin{tabular}{|c|c|c|c|c|c|c|}
\hline \multirow{2}{*}{ Firm A } & Firm B & \multicolumn{2}{|c|}{ Capture } & Loss & \multicolumn{2}{|c|}{ Capture } \\
\hline & & & & & & \\
3 & 4 & 1267 & $35 \%$ & $0 \%$ & 2308 & $65 \%$ \\
2 & 4 & 1062 & $30 \%$ & $16 \%$ & 2513 & $70 \%$ \\
1 & 4 & 852 & $24 \%$ & $33 \%$ & 2723 & $76 \%$ \\
\hline
\end{tabular}

Initially, before any of its outlets are to be closed, Firm A has 35\% of the market share. If the number of Firm A's outlets n reduced to 3, Firm A looses $16 \%$ of its market share to the competitor. If two outlets are eliminated, the loss is $33 \%$, and if three outlets are eliminated, Firm A owns only $13 \%$ of the market share. Results are presented graphically in Figure 1.

\section{Conclusions}

Models of facility closing are not well developed in the literature. We have proposed in this paper two new models that address facility closing. One of these investigates facility closing in a competitive environment, and the other examines closing in the situation of financial exigency without competitors. Application results for closing facilities in a competitive environment were presented to demonstrate the power of these models in such situations. 


\section{References}

J. Bruno and P. Anderson (1982): "Analytical methods for planning educational facilities in an era of declining enrolments", Socio-Economic Planning Sciences 16(3), 121-131.

Richard Church and Alan Murray (1993): "Modeling school utilization and consolidation", Journal of Urban Planning and Development 119(1), 23-38.

Richard Church and Charles ReVelle (1974): "The Maximal Covering Location Problem", Papers of the Regional Science Association, 32, 101 - 118.

J. Diamond and J. Wright (1987): "Multiobjective analysis of public school consolidation", Journal of Urban Planning and Development 113(1), 1-18.

John G. Klincewicz, Hanan Luss and Chang-Sung Yu (1988): "A large-scale multilocation capacity planning model", European Journal of Operation Research 34(2), 178-190.

Rein Lepnurm and Marje K. Lepnurm (2003), "The closure of rural hospitals in Saskatchewan: method or madness?" Social Science and Medicine 52(11), 16071707

Emanuel Melachrinoudis and Hokey Min (2000): "The dynamic relocation and phase-out of a hybrid, two-echelon plant/warehousing facility: A multiple objective approach", European Journal of Operational Research 123(1), 1-15.

Alan Murray and X. Wu (2003): "Accessibility tradeoffs in Public Transit Planning", Journal of Geographical Systems 5(1) 93-107.

Charles ReVelle (1986). "The Maximum capture or sphere of influence problem : Hotelling revisited on a network", Journal of Regional Science 26, 343-358.

Jarred Schenke (2002): "Keymart eyes more store closings", Atlanta Business Chronicle, Nov.4.

Qian Wang, Rajan Batta, Joyendu Badhury and Christopher Rump (2003): "Budget constrained location problem with opening and closing of facilities", Computers and Operations Research 30(13), 2047-2069.

Frederick C. Weston (1982), 'Optimal configuration of telephone answering sites in a service industry" European Journal of Operation Research 10(4), 395-405. 
Table A.1: 55-node network data

\begin{tabular}{|l|l|l|l|l|l|l|l|}
\hline NODE & POPN & COORD X & COORD Y & NODE & POPN & COORD X & COORD Y \\
\hline 1 & 710 & 32 & 31 & 29 & 60 & 19 & 38 \\
\hline 2 & 620 & 29 & 32 & 30 & 60 & 27 & 41 \\
\hline 3 & 560 & 27 & 36 & 31 & 60 & 21 & 35 \\
\hline 4 & 390 & 29 & 29 & 32 & 50 & 32 & 45 \\
\hline 5 & 350 & 32 & 29 & 33 & 50 & 27 & 45 \\
\hline 6 & 210 & 26 & 25 & 34 & 50 & 32 & 38 \\
\hline 7 & 200 & 24 & 33 & 35 & 50 & 8 & 22 \\
\hline 8 & 190 & 30 & 35 & 36 & 50 & 15 & 25 \\
\hline 9 & 170 & 29 & 27 & 37 & 50 & 35 & 16 \\
\hline 10 & 170 & 29 & 21 & 38 & 40 & 36 & 47 \\
\hline 11 & 160 & 33 & 28 & 39 & 40 & 46 & 51 \\
\hline 12 & 150 & 17 & 53 & 40 & 40 & 50 & 40 \\
\hline 13 & 140 & 34 & 30 & 41 & 40 & 23 & 22 \\
\hline 14 & 120 & 25 & 60 & 42 & 40 & 27 & 30 \\
\hline 15 & 120 & 21 & 28 & 43 & 40 & 38 & 39 \\
\hline 16 & 110 & 30 & 51 & 44 & 40 & 36 & 32 \\
\hline 17 & 100 & 19 & 47 & 45 & 30 & 32 & 41 \\
\hline 18 & 100 & 17 & 33 & 46 & 30 & 42 & 36 \\
\hline 19 & 90 & 22 & 40 & 47 & 30 & 36 & 26 \\
\hline 20 & 90 & 25 & 14 & 48 & 30 & 15 & 19 \\
\hline 21 & 90 & 29 & 12 & 49 & 30 & 19 & 14 \\
\hline 22 & 80 & 24 & 48 & 50 & 30 & 45 & 19 \\
\hline 23 & 80 & 17 & 42 & 51 & 30 & 27 & 5 \\
\hline 24 & 80 & 6 & 26 & 52 & 20 & 52 & 24 \\
\hline 25 & 80 & 19 & 21 & 53 & 20 & 40 & 22 \\
\hline 26 & 70 & 10 & 32 & 54 & 20 & 40 & 52 \\
\hline 27 & 60 & 34 & 56 & 55 & 20 & 42 & 42 \\
\hline 28 & 60 & 12 & 47 & & & & \\
\hline & & & & & & \\
\hline
\end{tabular}

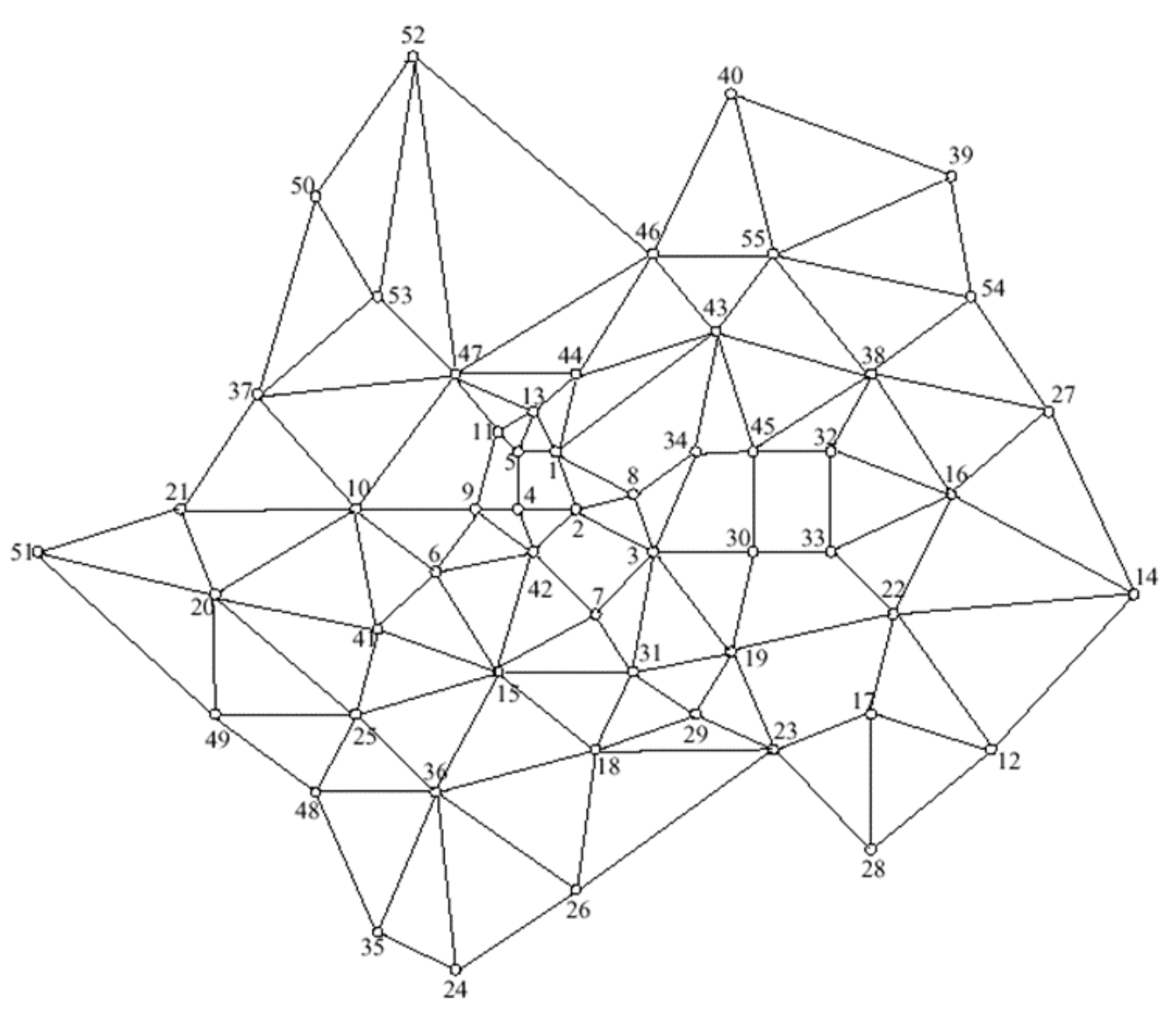


Figure 1: results, 55-node network
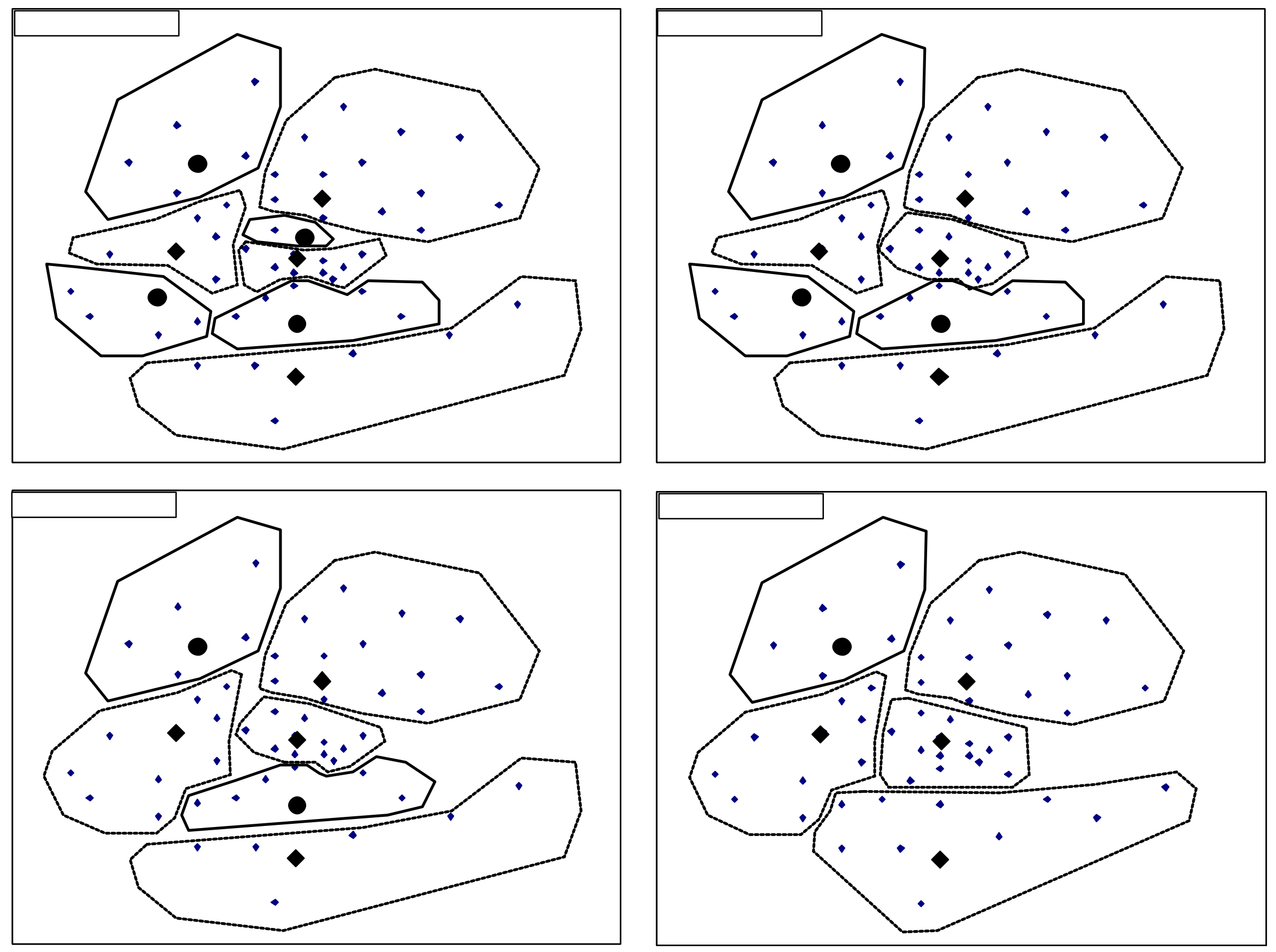